Article

\title{
An Electrochemical Method to Detect Gamma Glutamyl Transpeptidase
}

\section{Guifang Chen ${ }^{1}$, Shengfa $\mathrm{Ni}^{2}$, Sha Zhu ${ }^{2}$, Jinghua Yang ${ }^{3}$ and Yongmei Yin ${ }^{2, *}$}

1 Laboratory of Biosensing Technology, School of Life Sciences, Shanghai University, Shanghai 200444, China; E-Mail: gfchen@shu.edu.cn

2 Department of Oncology, the First Affiliated Hospital of Nanjing Medical University, Nanjing 210029, China; E-Mails: inzo@163.com (S.N.); nanyizhusha@126.com (S.Z.)

3 Department of Biochemistry and State Key Laboratory of Pharmaceutical Biotechnology, Nanjing University, Nanjing 210093, China; E-Mail: josephinenju@yahoo.com.cn

* Author to whom correspondence should be addressed; E-Mail: YMYin2011@gmail.com; Tel.: +86-25-68136043; Fax: +86-25-83710040.

Received: 31 December 2011; in revised form: 3 February 2012 / Accepted: 27 February 2012 / Published: 2 March 2012

\begin{abstract}
Gamma glutamyl transpeptidase (GGT) is a transferase, which is of great importance in sustaining intracellular cysteine and glutathione levels. The abnormal expression of GGT is significantly associated with features of many metabolic syndromes (e.g., hepatocellular carcinoma). Therefore, it is essential to develop methods to detect GGT so as to monitor the physiological or pathological phenomena related to this species. In this work, by making use of a complex formed by $\mathrm{Cu}^{2+}$ and glutathione, which may exhibit excellent voltammetric response, we have proposed a novel potential electrochemical method for the detection of the enzyme. Results show that in the presence of GGT, the formation of $\mathrm{Cu}^{2+}$-glutathione complex on a working electrode will be disrupted, resulting in greatly depressed electrochemical signals. The primary method exhibits some advantages, such as it being fast, cost-efficient, and conveniently operated. It also has the potential to be further developed as an effective method in the quantitative detection of GGT in real samples.
\end{abstract}

Keywords: gamma glutamyl transpeptidase; glutathione; copper ions; electrochemical 


\section{Introduction}

Gamma glutamyl transpeptidase (GGT) is a cell membrane-bound enzyme, which is located on the outer surface of cell membranes. It catalyzes the transfer of the gamma-glutamyl moiety of glutathione (GSH) to an amino acid, a peptide or water [1]. GSH, a tripeptide consisting of glutamine, cysteine and glycine, is produced in almost all cells. It is widely distributed in various tissues and performs many important physiological functions [2,3]. GGT plays a key role in the metabolism of GSH, and significantly contributes to sustaining intracellular cysteine and glutathione levels [4]. It is also involved in cancer drug resistance when overexpressed [5,6], and the over expression may also provide cancer cells with survival advantages under stress conditions [7]. Moreover, GGT-mediated metabolism of extracellular GSH is an important source of cysteine for tumor cells to resynthesize GSH. The increased expression of GGT is generally interpreted as a factor that favors the growth of several tumors [8]. Therefore, elevation of GGT is significantly associated with the features of many metabolic syndromes [9], and the detection of the enzyme may have important clinical applications. For example, hepatocellular carcinoma (HCC), which is frequently the long term result of viral hepatitis $\mathrm{B}(\mathrm{HBV})$ or $\mathrm{C}(\mathrm{HCV})$ infections associated with chronic inflammation and cirrhosis, is one of the most common fatal malignancies in the world. About 90 percent of tumors originating from liver are HCC. Although the diagnosis and treatment methods of HCC have been developing over the last 20 years, the recurrence and mortality of HCC is still high. One reason is that patients with HCC in the early stage are asymptomatic, and most of them are perhaps in the late-stage, advanced, or micro metastasis state when they are diagnosed [10]. So, early diagnosis is as important as efficient treatment and the understanding of the pathogenic mechanism of HCC.

It is known that some serum inflammatory markers are strongly related to HCC prognosis [11-15]; GGT is one of them, and has been considered for the evaluation of chronic hepatitis activity [16]. Some studies from HBV or HCV-related HCC have suggested that high levels of GGT are related to high incidence of HCC development, recurrence and poor prognosis [17]. Patients with HCC or some other hepatobiliary diseases are usually diagnosed to have high levels of GGT in their serum [18,19]. The serum activity of GGT may be even used as a diagnostic marker of liver cancer [20]. Owing to the importance of GGT, some available methods have been developed to detect the enzyme. Spectrophotometric measurement [21,22], which is recommended by the International Federation for Clinical Chemistry and Laboratory Medicine (IFCC), is the most prevailing method, and has been adopted in clinics. Besides fluorescence [23], high-performance liquid chromatography (HPLC) [24], and electrophoresis [25] have also been successfully employed in the detection of the activity of GGT. Though the quantitative detection of GGT in vitro has thereby been achieved, some disadvantages still exist. For example, electrophoresis and HPLC methods are relatively time consuming and inconvenient, while spectrophotometric and fluorescent measurements need costly apparatus and some toxic chemical reagents (e.g., 5-amino-2-nitrobenzoate), which restrict the development of portable home medical devices. Electrochemical technique has been well known for its fast detection, easy operation, low cost, high sensitivity, and easy-to-miniaturize. In this work, we propose a novel primary electrochemical method to detect GGT, which may be further developed as an effective method in the quantitative detection of GGT in real samples. 


\section{Results and Discussion}

We first immobilized the substrate GSH onto a gold electrode through an Au-S bond between the surface of electrode and the thiol group of GSH. On the other hand, copper ions have been proven to exhibit electrochemical signals by its complexation with some species, such as GSH [26], polyaspartate [27] and cysteine [28]. This finding is also confirmed by our results. As is shown in Figure 1, after complexation with $\mathrm{Cu}^{2+}$, the GSH modified electrode may show voltammetric peaks, whereas no redox waves can be observed in the absence of $\mathrm{Cu}^{2+}$. The redox peaks are attributed to the reduction and oxidation of $\mathrm{Cu}^{2+} / \mathrm{Cu}^{+}$couples. Further results show that after the GSH modified electrode is treated with GGT, the voltammetric peaks of the electrode decrease.

Figure 1. Cyclic voltammograms obtained at the glutathione (GSH) modified electrode before (dot line) and after (solid line) it is treated with $1 \mu \mathrm{M} \mathrm{Cu}^{2+}$ for $1 \mathrm{~h}$. The dash line shows the case that the GSH modified electrode has been previously incubated with $5 \mathrm{U} / \mathrm{mL} \mathrm{GGT}$ at $37^{\circ} \mathrm{C}$ for $30 \mathrm{~min}$ before the treatment of $\mathrm{Cu}^{2+}$. Scan rate: $50 \mathrm{mV} \cdot \mathrm{s}^{-1}$.

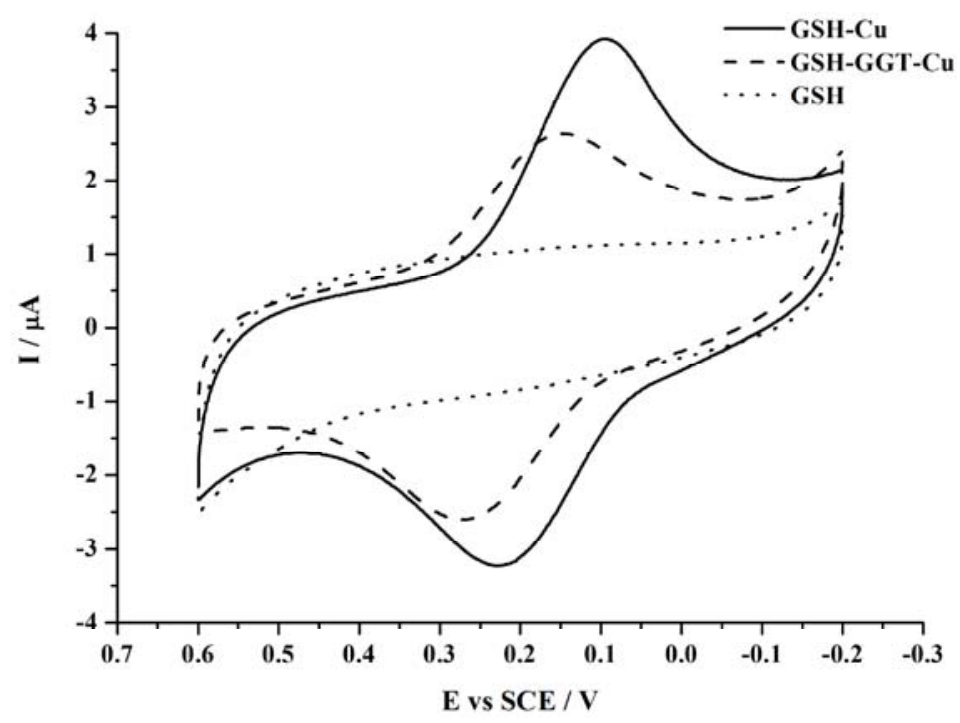

The result is as expected, because under the catalysis of GGT, the glutamic acid moiety of GSH is cut off, leaving the cysteinylglycine moiety alone attached on the electrode, which cannot then form a complex with $\mathrm{Cu}^{2+}$. Therefore, after the incubation of the GSH modified electrode with the enzyme, the amount of $\mathrm{Cu}^{2+}$-GSH complex decreases, which results in the decreased electrochemical peaks.

Electrochemical impedance spectrum (EIS) technique is employed to characterize the surface alteration of the gold electrode after its modification with GSH, and subsequently the catalysis by GGT. Because GSH is unfavorable for the electron transfer between the electrochemical probe $\left[\mathrm{Fe}(\mathrm{CN})_{6}\right]^{3-/ 4-}$ and the electrode surface as a result of steric exclusion, after the modification of GSH onto the gold electrode surface, an increase of the electron-transfer resistance can be observed (Figure 2), representing a big semi-circle. However, after the catalysis by GGT, the glutamic acid moiety of GSH is cut off from the electrode and the steric exclusion decrease, representing a small semi-circle, whose diameter is between a GSH modified electrode and a bare electrode. 
Figure 2. Electrochemical impedance spectra of GSH modified electrode: (a) before; (b) after its incubation with $5 \mathrm{U} / \mathrm{mL}$ GGT at $37{ }^{\circ} \mathrm{C}$ for $30 \mathrm{~min}$. Curve (c) is in the case of bare gold electrode. $5 \mathrm{mM} \mathrm{K}_{3} \mathrm{Fe}(\mathrm{CN})_{6} / \mathrm{K}_{4} \mathrm{Fe}(\mathrm{CN})_{6}$ working as electrochemical probe was added to the electrolyte, initial E was set at $0.224 \mathrm{~V}$, amplitude was $0.005 \mathrm{~V}$, high and low frequency were $10^{5} \mathrm{~Hz}$ and $0.01 \mathrm{~Hz}$, respectively.

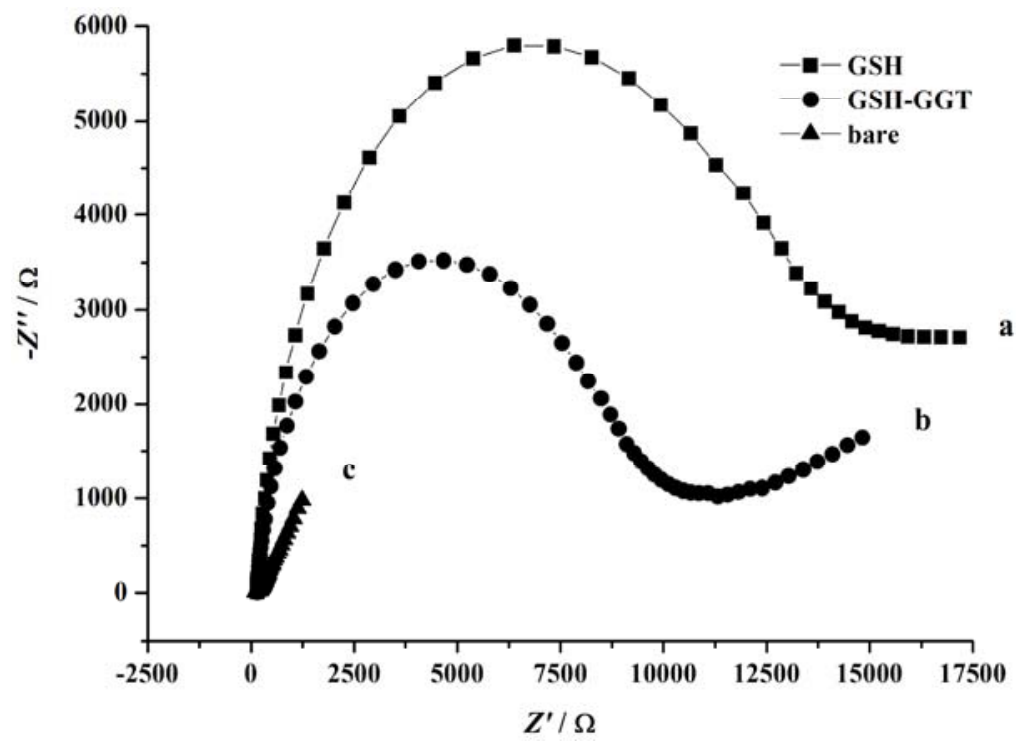

Figure 3. Cyclic voltammograms obtained at the GSH modified electrode in the presence of $1 \mu \mathrm{M} \mathrm{Cu}{ }^{2+}$ for $1 \mathrm{~h}$. The GSH has been pre-incubated with $5 \mathrm{U} / \mathrm{mL}$ GGT at $37{ }^{\circ} \mathrm{C}$ (dash dot line) or $25{ }^{\circ} \mathrm{C}$ (dash line) for $30 \mathrm{~min}$, or without GGT (solid line), and then incubated with a gold electrode for $16 \mathrm{~h}$ to form the GSH modified electrode. The dot line shows the case that EDTA instead of GGT is added in the test solution. Scan rate: $50 \mathrm{mV} \cdot \mathrm{s}^{-1}$.

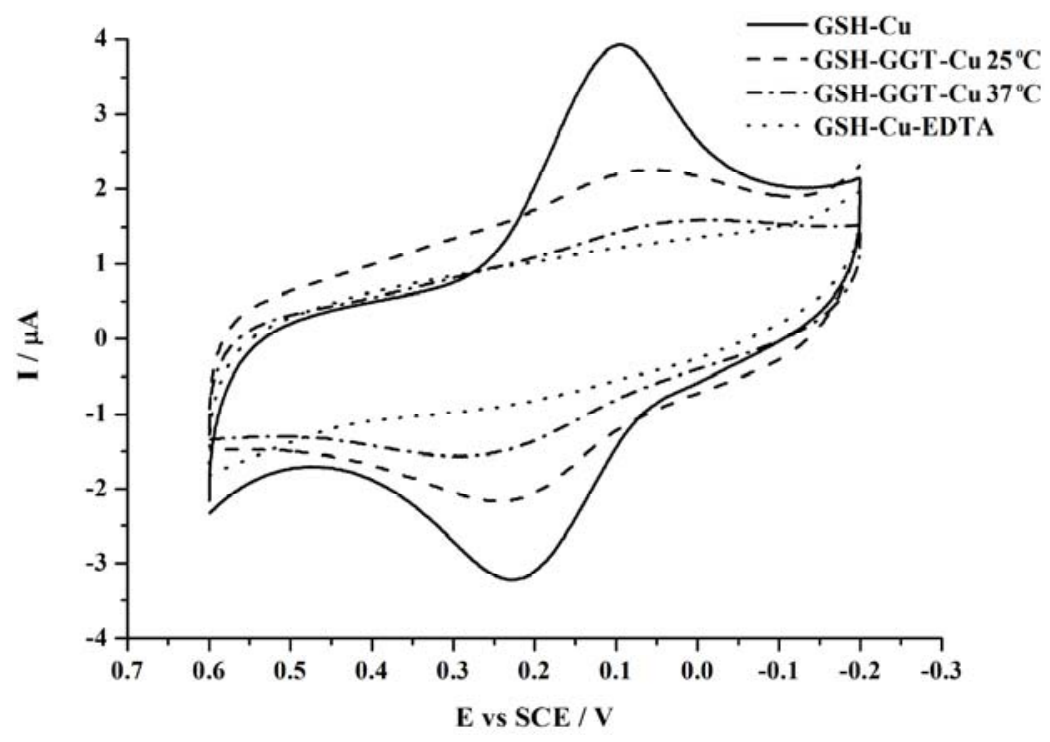

We also conducted the experiments using an alternative strategy in which the enzyme catalyzed reaction takes place in solution rather than on the electrode surface. As has been described in the experimental sections, GGT is first allowed to catalyze the transfer of the glutamic acid moiety of GSH in a solution. The production containing glutamic acid and cysteinylglycine together with the 
enzyme itself is then incubated with a polished electrode, allowing the interaction between cysteinylglycine and the surface of the electrode. After further treatment with $\mathrm{Cu}^{2+}$, the electrode is measured by cyclic voltammetry (CV). As is shown in Figure 3, the peaks decrease more drastically than that in the former case (dash dot line in Figure 3 compared with dash line in Figure 1). The result is reasonable, since the solution environment provides more opportunities for the interaction between GGT and GSH than that on a surface, and most of GSH is cut off, consequently less $\mathrm{Cu}^{2+}-\mathrm{GSH}$ complex can be formed to provide electrochemical signals.

Besides, we conduct the experiments at $25{ }^{\circ} \mathrm{C}$ to further confirm that the current decrease is attributed to the GGT catalysis. As is also shown in Figure 3 (dash line), the current decrease is less prominent than that at $37^{\circ} \mathrm{C}$. Because the optimum temperature for GGT is $37^{\circ} \mathrm{C}$, the activity of GGT is limited at $25^{\circ} \mathrm{C}$, so as to show an expected experimental performance.

As is well known, $\mathrm{Cu}^{2+}$ is chelated with EDTA very strongly. So we make use of EDTA as an inhibitor for the formation of $\mathrm{Cu}^{2+}-\mathrm{GSH}$ complex. As is shown in Figure 3 (dot line), while EDTA instead of GGT is added, the current decrease is so apparent that no peaks can be observed anymore, suggesting that the chelation between $\mathrm{Cu}^{2+}$ and EDTA is more efficient than the catalysis of GGT.

Scheme 1. Schematic illustration of the electrochemical method to detect the activity of GGT. (A) A GSH modified electrode is first prepared. Then the GGT-mediated catalysis is allowed to occur on the surface of the electrode. Finally, $\mathrm{Cu}^{2+}$ is added in order to produce the electrochemical response; (B) GSH is first cleaved by GGT in a solution. Then GSH is linked to a gold electrode. Finally, $\mathrm{Cu}^{2+}$ is added in order to produce the electrochemical response.

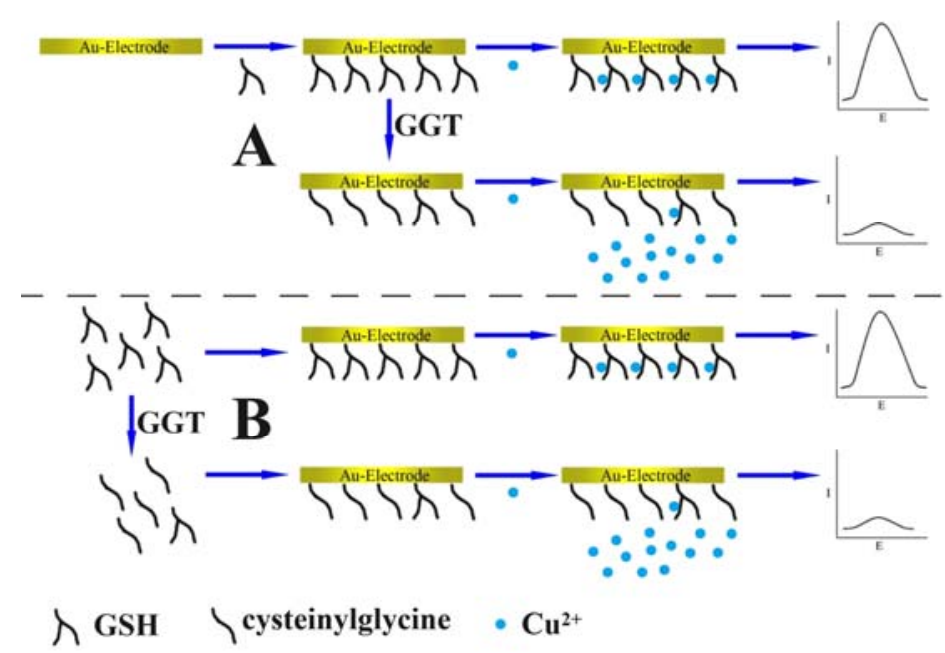

The overall method for the detection of GGT is illustrated in Scheme 1. As is shown in this scheme, two strategies with slight differences are proposed. In Scheme 1A, GGT-mediated catalysis occurs on the surface of the electrode, while in Scheme 1B, the catalytic reaction takes place in the solution. Despite the difference, results show that both of the strategies work well. In the former case, the strategy is more convenient to operate, because only ca. $90 \mathrm{~min}$ is required to complete the detection at the introduction of the target GGT. The latter is otherwise better in signal behavior, since the GGT caused current changes are more drastic in this case. The choice of which one is better should depend on the needs and further tests. In comparison with the prevailing spectrophotometric method, the 
electrochemical method shown here has comparative operability, test time, and cost. A main disadvantage of the electrochemical method is that a modified electrode should be prepared, which requires hours (16 $\mathrm{h}$ in our experiments). Fortunately, it may be excluded in the test process (strategy shown in Schme 1A) and may be commercially mass-produced. On the other hand, the major advantage of the electrochemical method is that all the experimental materials are readily obtained and cost-effective without requiring the usage of toxic chemical reagents.

\section{Experimental Section}

\subsection{Materials}

GGT from equine kidney (9.1 mg, 100 Units) was purchased from Sigma and used without further purification. GSH was purchased from Nanjing Dazhi Biotechnology Co., Ltd. $\mathrm{CuSO}_{4}$, and EDTA were acquired from Nanjing Chemical Reagent Co., Ltd, which were both of analytical grade. $\mathrm{CuSO}_{4}$ was dissolved in $0.1 \mathrm{mM} \mathrm{HNO}_{3}$. All solutions were prepared with double-distilled water which was purified to a specific resistance $(18.2 \mathrm{M} \Omega / \mathrm{cm})$ with a Milli-Q purification system (Barnstead, Asheville, $\mathrm{NC}, \mathrm{USA}$ ) and were stored in $4{ }^{\circ} \mathrm{C}$ for use.

\subsection{Preparation of GSH Modified Electrode}

Gold electrode, the gold diameter of which was three millimeters, was purchased from Ada Tianjin Science and Technology Development Co., Ltd. The gold electrode was firstly polished on fine sand papers and alumina (particle size of about $0.05 \mu \mathrm{m}$ )/water slurry on silk. Then it was thoroughly ultrasonicated in ethanol and doubly distilled water for about $5 \mathrm{~min}$, respectively. Finally, the electrode was electrochemically cleaned to remove any remaining impurities in $1 \mathrm{M} \mathrm{H}_{2} \mathrm{SO}_{4}$. After being dried with nitrogen, the gold electrode was immersed in $0.5 \mathrm{~mL}$ solution containing $3 \mathrm{mg} / \mathrm{mL}$ GSH for $16 \mathrm{~h}$. The GSH could bind to the gold electrode through an Au-S bond between the surface of electrode and the thiol group of GSH. After thoroughly rinsed with pure water and dried with nitrogen, GSH modified electrode was prepared and could be ready for use.

\subsection{GGT Catalyzed Reaction on the GSH Modified Electrode}

The prepared GSH modified gold electrode was further immersed in a $0.5 \mathrm{~mL}$ solution containing $5 \mathrm{U} / \mathrm{mL}$ GGT at $37^{\circ} \mathrm{C}$ for $30 \mathrm{~min}$, during which the GGT would catalyze the transfer of the glutamic acid moiety of GSH, leaving the cysteinylglycine moiety alone attached on the electrode. After thoroughly rinsed with pure water, cysteinylglycine modified electrode was prepared. For comparison, the cysteinylglycine modified electrode could be also prepared by an alternative strategy, in which the enzyme catalyzed reaction took place in solution. In this strategy, a solution containing $5 \mathrm{U} / \mathrm{mL}$ GGT and $3 \mathrm{mg} / \mathrm{mL}$ GSH was first incubated at $37^{\circ} \mathrm{C}$ for $30 \mathrm{~min}$ to allow the reaction occur. Then, a polished gold electrode was immersed in the solution for $16 \mathrm{~h}$. After being thoroughly rinsed with pure water, the cysteinylglycine modified electrode was prepared. 


\subsection{Electrochemical Measurements}

The GSH modified electrode or cysteinylglycine modified electrode was further immersed in a solution containing $1 \mu \mathrm{M} \mathrm{CuSO}_{4}$ for an hour before electrochemical measurements. Then, the electrode was thoroughly rinsed with pure water, and was ready for measurements. Electrochemical measurements by using $\mathrm{CV}$ and EIS techniques were performed on a model 660C Electrochemical Analyzer $(\mathrm{CH}$ Instruments) with a PBS buffer (100 mM, pH 7.0) working as electrolyte. The modified electrode was used as working electrode, and a saturated calomel electrode (SCE) and a platinum electrode were used as the reference and counter electrode, respectively. For CV test, scan rate was set at $50 \mathrm{mV} / \mathrm{s}$, while for EIS, $5 \mathrm{mM} \mathrm{K}{ }_{3} \mathrm{Fe}(\mathrm{CN})_{6} / \mathrm{K}_{4} \mathrm{Fe}(\mathrm{CN})_{6}$ working as electrochemical probe was added to the electrolyte, initial $\mathrm{E}$ was set at $0.224 \mathrm{~V}$, amplitude was $0.005 \mathrm{~V}$, high and low frequency were $10^{5} \mathrm{~Hz}$ and $0.01 \mathrm{~Hz}$, respectively.

\section{Conclusions}

In summary, we have developed an electrochemical method to detect GGT based on the voltammetric response of the complex formed by $\mathrm{Cu}^{2+}$ and $\mathrm{GSH}$. The detection can be easily and conveniently performed, and the preparation of the modified electrode is also simple without any troublesome procedures necessary. Therefore, the proposed method for the detection of GGT may have potential application in the diagnosis of liver cancer in the future.

\section{Acknowledgments}

This work is supported by the National Science Fund for Distinguished Young Scholars (Grant No. 20925520), the Leading Academic Discipline Project of Shanghai Municipal Education Commission (J50108), and the Shanghai Science and Technology Committee No. 11DZ2272100.

\section{References}

1. Griffith, O.W.; Bridges, R.J.; Meister, A. Transport of gamma-glutamyl amino acids: Role of glutathione and gamma-glutamyl transpeptidase. Proc. Natl. Acad. Sci. USA 1979, 76, 6319-6322.

2. Meister, A.; Anderson, M.E. Glutathione. Annu. Rev. Biochem. 1983, 52, 711-760.

3. Pero, M.E.; Pelagalli, A.; Lombardi, P.; Avallone, L. Glutathione concentration and gamma-glutamyltransferase activity in water buffalo colostrums. J. Anim. Physiol. Anim. Nutr. 2010, 94, 549-551.

4. Jenderny, S.; Lin, H.; Garrett, T.; Tew, K.D.; Townsend, D.M. Protective effects of a glutathione disulfide mimetic (NOV-002) against cisplatin induced kidney toxicity. Biomed. Pharmacother. 2010, 64, 73-76.

5. Reuter, S.; Schnekenburger, M.; Cristofanon, S.; Buck, I.; Teiten, M.H.; Daubeuf, S.; Eifes, S.; Dicato, M.; Aggarwal, B.B.; Visvikis, A.; et al. Tumor necrosis factor $\alpha$ induces $\gamma$-glutamyltransferase expression via nuclear factor- $\mathrm{\kappa B}$ in cooperation with $\mathrm{Sp} 1$. Biochem. Pharmacol. 2009, 77, 397-411.

6. Pompella, A.; de Tata, V.; Paolicchi, A.; Zunino, F. Expression of $\gamma$-glutamyltransferase in cancer cells and its significance in drug resistance. Biochem. Pharmacol. 2006, 71, 231-238. 
7. Giommarelli, C.; Corti, A.; Supino, R.; Favini, E.; Paolicchi, A.; Pompella, A.; Zunino, F. Cellular response to oxidative stress and ascorbic acid in melanoma cells overexpressing $\gamma$-glutamyltransferase. Eur. J. Cancer 2008, 44, 750-759.

8. Pompella, A.; Corti, A.; Paolicchi, A.; Giommarelli, C.; Zunino, F. $\gamma$-Glutamyltransferase, redox regulation and cancer drug resistance. Curr. Opin. Pharmacol. 2007, 7, 360-366.

9. Whitfield, J.B. Gamma glutamyl transferase. Crit. Rev. Clin. Lab. Sci. 2001, 38, 263-355.

10. Hann, H.W.L.; Lee, J.; Bussard, A.; Liu, C.; Jin, Y.R.; Guha, K.; Clayton, M.M.; Ardlie, K.; Pellini, M.J.; Feitelson, M.A. Preneoplastic markers of hepatitis B virus-associated hepatocellular carcinoma. Cancer Res. 2004, 64, 7329-7335.

11. Gao, Q.; Qiu, S.J.; Fan, J.; Zhou, J.; Wang, X.Y.; Xiao, Y.S.; Xu, Y.; Li, Y.W.; Tang, Z.Y. Intratumoral balance of regulatory and cytotoxic $\mathrm{T}$ cells is associated with prognosis of hepatocellular carcinoma after resection. J. Clin. Oncol. 2007, 25, 2586-2593.

12. Infante, J.R.; Matsubayashi, H.; Sato, N.; Tonascia, J.; Klein, A.P.; Riall, T.A.; Yeo, C.; Iacobuzio-Donahue, C.; Goggins, M. Peritumoral fibroblast SPARC expression and patient outcome with resectable pancreatic adenocarcinoma. J. Clin. Oncol. 2007, 25, 319-325.

13. Tarao, K.; Takemiya, S.; Tamai, S.; Sugimasa, Y.; Ohkawa, S.; Akaike, M.; Tanabe, H.; Shimizu, A.; Yoshida, M.; Kakita, A. Relationship between the recurrence of hepatocellular carcinoma (HCC) and serum alanine aminotransferase levels in hepatectomized patients with hepatitis C virus-associated cirrhosis and HCC. Cancer 1997, 79, 688-694.

14. Nagasue, N.; Kohno, H.; Tachibana, M.; Yamanoi, A.; Ohmori, H.; El-Assal, O.N. Prognostic factors after hepatic resection for hepatocellular carcinoma associated with child-turcotte class B and C cirrhosis. Ann. Surg. 1999, 229, 84-90.

15. Zhou, X.D.; Tang, Z.Y.; Yang, B.H.; Lin, Z.Y.; Ma, Z.C.; Ye, S.L.; Wu, Z.Q.; Fan, J.; Qin, L.X.; Zheng, B.H. Experience of 1000 patients who underwent hepatectomy for small hepatocellular carcinoma. Cancer 2001, 91, 1479-1486.

16. Silva, I.S.; Ferraz, M.L.; Perez, R.M.; Lanzoni, V.P.; Figueiredo, V.M.; Silva, A.E. Role of $\gamma$-glutamyl transferase activity in patients with chronic hepatitis $\mathrm{C}$ virus infection. J. Gastroenterol. Hepatol. 2004, 19, 314-318.

17. Yao, D.F.; Jiang, D.R.; Huang, Z.W.; Lu, J.X.; Tao, Q.Y.; Yu, Z.J.; Meng, X.Y. Abnormal expression of hepatoma specific $\gamma$-glutamyl transferase and alteration of $\gamma$-glutamyl transferase gene methylation status in patients with hepatocellular carcinoma. Cancer 2000, 88, 761-769.

18. Stefaniuk, P.; Cianciara, J.; Wiercinska-Drapalo, A. Present and future possibilities for early diagnosis of hepatocellular carcinoma. World J. Gastroenterol. 2010, 16, 418-424.

19. Ikeda, Y.; Taniguchi, N. Gene expression of $\gamma$-glutamyltranspeptidase. Methods Enzymol. 2005, 401, 408-425.

20. Pettersen, I.; Andersen, J.H.; Bjornland, K.; Mathisen, O.; Bremnes, R.; Wellman, M.; Visvikis, A.; Huseby, N.E. Heterogeneity in $\gamma$-glutamyltransferase mRNA expression and glycan structures. Search for tumor-specific variants in human liver metastases and colon carcinoma cells. Biochim. Biophys. Acta 2003, 1648, 210-218.

21. Huseby, N.E.; Stromme, J.H. Practical points regarding routine determination of $\gamma$-glutamyl transferase $(\gamma-\mathrm{GT})$ in serum with a kinetic method at $37^{\circ} \mathrm{C}$. Scand. J. Clin. Lab. Invest. 1974, 34, $357-361$. 
22. Masia, A.; Destroa, T.; Turettaa, L.; Varottob, S.; Caporalec, G.; Ferrettia, M. Localization of $\gamma$-glutamyl transferase activity and protein in Zea mays organs and tissues. J. Plant Physiol. 2007, 164, 1527-1535.

23. Urano, Y.; Sakabe, M.; Kosaka, N.; Ogawa, M.; Mitsunaga, M.; Asanuma, D.; Kamiya, M.; Young, M.R.; Nagano, T.; Choyke, P.L.; et al. Rapid cancer detection by topically spraying a $\gamma$-glutamyltranspeptidase-activated fluorescent probe. Sci. Transl. Med. 2011, 3, doi:10.1126/scitranslmed.3002823.

24. Kiuchi, K.; Kiuchi, K.; Nagatsu, T.; Togari, A.; Kumagai, H. Highly sensitive assay for $\gamma$-glutamyltranspeptidase activity by high-performance liquid chromatography with electrochemical detection. J. Chromatogr. 1986, 357, 191-198.

25. Yao, D.F.; Huang, Z.W.; Chen, S.Z.; Huang, J.F.; Lu, J.X.; Xiao, M.B.; Meng, X.Y. Diagnosis of hepatocellular carcinoma by quantitative detection of hepatoma-specific bands of serum

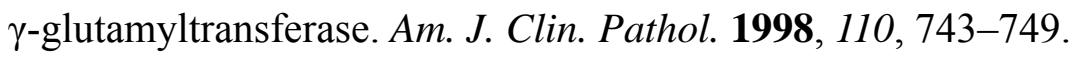

26. Fang, C.; Zhou, X.Y. Voltammetry and EQCM investigation of glutathione monolayer and its complexation with $\mathrm{Cu}^{2+}$. Electroanalysis 2003, 15, 20-26.

27. Yang, W.R.; Gooding, J.J.; Hibbert, D.B. Redox voltammetry of sub-parts per billion levels of $\mathrm{Cu}^{2+}$ at polyaspartate-modified gold electrodes. Analyst 2001, 126, 1573-1577.

28. Liu, A.C.; Chen, D.C.; Lin, C.C.; Chou, H.H.; Chen, C.H. Application of cysteine monolayers for electrochemical determination of sub-ppb copper(II). Anal. Chem. 1999, 71, 1549-1552.

(C) 2012 by the authors; licensee MDPI, Basel, Switzerland. This article is an open access article distributed under the terms and conditions of the Creative Commons Attribution license (http://creativecommons.org/licenses/by/3.0/). 